\title{
41
}

\section{Light-sheet flow cytometry}

Sonia Laguna $^{1}$, Clara Gómez-Cruz ${ }^{1}$, Ignacio Albert-Smet ${ }^{1}$, Juan José Vaquero $^{1,2}$, Jorge Ripoll ${ }^{1,2}$, Arrate Muñoz-Barrutia ${ }^{1,2}$

${ }^{1}$ Departamento de Bioingeniería e Ingeniería Aeroespacial, Universidad Carlos III de Madrid, Spain. ${ }^{2}$ Instituto de Investigación Sanitaria del Hospital Gregorio Marañón, Spain

\section{Abstract Text}

Introduction: Flow cytometry is a laser-based technology used to quantify, sort, and analyze particles in a solution [1]. This work presents a custom-made micro-flow cytometry platform to quantify Mycobacterium tuberculosis (Mtb), the agent that causes tuberculosis, using light-sheet fluorescence microscopy (LSFM). This project aims to quantify the number of living bacteria remaining in a solution after exposure to new potential compounds, as part of an effort to reduce the time burden of developing new antibiotics against $M t b$.

Methods: The light-sheet flow cytometer is composed of a microflow cytometry platform and a single-plane illumination microscopy (SPIM) system. This SPIM is characterized by two Galvo motors, which allow for 3D scanning of the sample and shadow reduction without moving the sample stage, avoiding unnecessary forces acting on the cytometry platform. Two electro-tunable lenses allow fine focusing from the software, improving the acquisition focus and 3D scanning control system by coordinating the laser movement and focal point. The configuration is shown in Figure 1. 

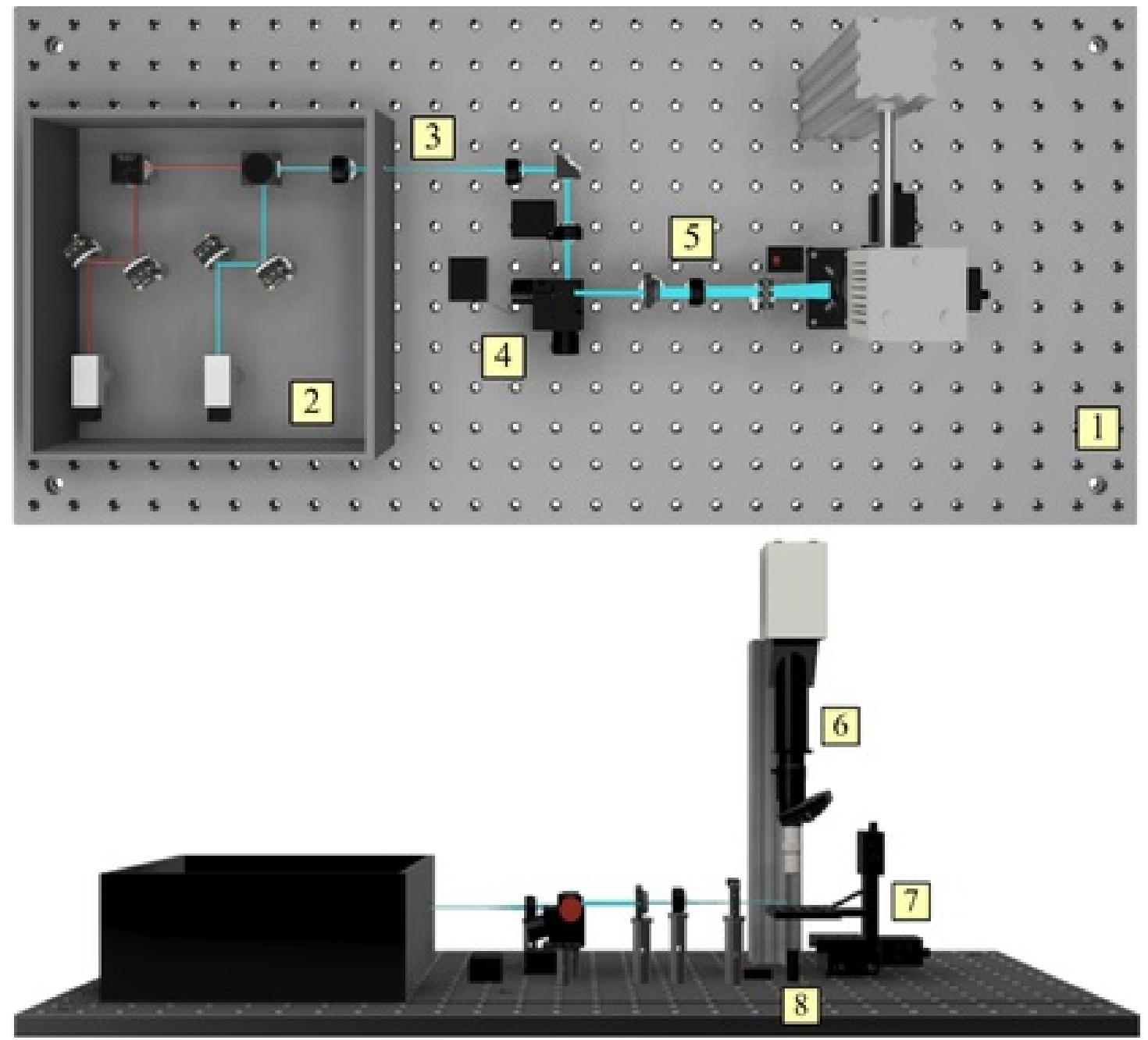

Figure 1. Fusion 360 model of the SPIM set up and components. (1) Optical table. (2) Lasers. (3) Lenses in beam expansion. (4) Galvo system, composed of a kinematic mirror, the first tunable lens, and a galvanometer. (5) Lenses creating the light-sheet. (6) Camera turret with components such as the objective, second tunable lens, and camera. (7) Linear stages and sample holding platform. (8) LED.

Likewise, microfluidic devices are used in the cytometry platform for the particle solution to flow in a controlled manner within the microscope. The microfluidic platform used is based on a design by Mao et al. [2] that achieves a 3D hydrodynamic focusing using a Dean vortex, ensuring that particles are focused in 3D within the channel for a successful discretization and quantification. An imageprocessing pipeline has been developed to detect particles flowing through the microfluidic channel in SPIM frames, with steps shown in Figure 2 . 


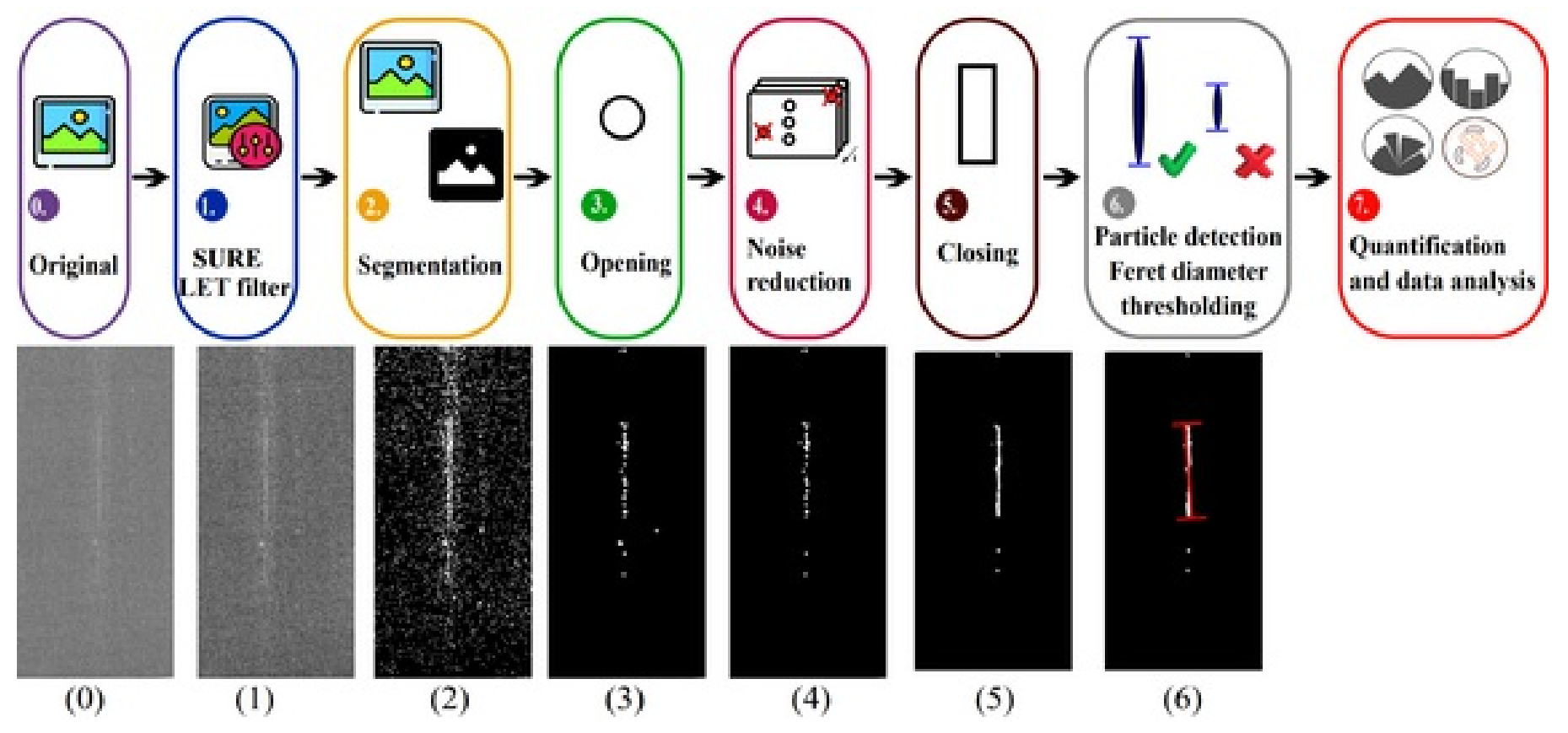

Figure 2. Image-processing pipeline with each step and example frames: (1) SURE-LET filtering, (2) thresholding segmentation, (3) opening with circular structural element, (4) noise reduction with previous frame, (5) closing with rectangular structural element, (6) particle detection and Feret diameter thresholding, (7) quantization and data analysis.

Results: The results illustrate success in the SPIM set up and adaptation into a flow cytometry platform, with a lateral resolution of $2.64 \mu \mathrm{m}$ and $34.19 \mu \mathrm{m}$ in the axial direction. The analysis of the image-processing carried out to quantify the particles from the initial experiments lead to a $94 \%$ sensitivity, suggesting an adequate operation of the pipeline.

Conclusion: This project shows that LSFM techniques and particularly SPIM are fit to be adapted into a flow-cytometer. The proposed platform successfully quantifies the number of particles flowing in a solution inside a microfluidic device and could potentially replace traditional methods for Mtb bacterial quantification.

Funding: Innovative Medicines Initiative (www.imi.europa.eu) Joint Undertaking AMR Accelerator programme, under grant agreement no. 853989 - ERA4TB, whose resources comprise funding from the European Union Horizon 2020 and EFPIA companies in-kind contribution; Project PID2019-109820RB-I00 from the Spanish Ministry of Science and Innovation. 
